SCIENTIFIC LETTER

\title{
Quality of life assessment using the short form 12 questionnaire is as reliable and sensitive as the short form 36 in distinguishing symptom severity in myocardial infarction survivors
}

\author{
M R Melville, M A Lari, N Brown, T Young, D Gray
}

Heart 2003;89:1445-1446

$\mathrm{F}$ rom the patient's perspective, disease severity is gauged by its impact on health related quality of life (HRQL), whereas medical practitioners tend to focus on clinical parameters such as symptoms, signs, and investigations. HRQL assessment can complement clinical evaluation but has not been widely incorporated into routine clinical practice, perhaps because of an association with research, lack of familiarity, difficulties with data interpretation, or concern that outpatients is busy enough.

The short form 36 questionnaire (SF-36) ${ }^{1}$ is an established HRQL tool that has been evaluated in a range of medical conditions $^{2}$ and for which normative data are available. ${ }^{3}$ Survivors of myocardial infarction have notably impaired HRQL compared with a normal population, detected more readily by the SF-36 than the Nottingham health profile (NHP). ${ }^{4}$ Despite being relatively user friendly, the SF-36 can be time consuming, especially when combined with essential demographic and other questionnaires, leading to reduced response rates, incompleteness, and unreliability.

We investigated whether a shorter questionnaire, the short form 12 (SF-12), which derives summary scores from specific items from the eight domains of the SF-36, might provide equally reliable and sensitive information in a post-infarction population.

\section{METHOD}

The patient population has been described before. ${ }^{4}$ Briefly, we mailed a detailed questionnaire comprising demographic questions, Rose angina and dyspnoea scales, SF-36, and NHP to all patients who had: (1) been admitted in a single year to Nottingham's two hospitals; (2) sustained an acute myocardial infarction, based on World Health Organization criteria; and (3) survived at least four years. HRQL scores for the SF12 were calculated.

\section{RESULTS}

Two hundred and five of 960 confirmed infarctions died in hospital; a further 210 died over the next four years. Those with recurrent infarctions or resident outside our health district were excluded, leaving 476 study patients.

\section{Response rates}

A total of 424 (89\%) patients responded; 421 (99\%) answered some or all of the SF-36, making it possible to calculate physical and mental summary scores for 304 (72\%). Based on mandated questions within the SF-36, equivalent SF-12 summary scores were calculated for 286 (68\%); 278 (66\%) had physical and mental summary scale scores for both the SF-36 and the SF-12.

\section{Patient characteristics}

There were no significant differences in the demographic characteristics of responders and non-responders. The mean (SD) age of those for whom SF-36 and SF-12 summary scores were available was 64 (9.97) years. One hundred and ninety eight $(71 \%)$ were male, 231 (83\%) had a Q wave infarction, and 108 (39\%) an anterior infarction; 192 (69\%) were Killip class 0 on admission, and $29(10 \%)$ had had a previous myocardial infarction. When the questionnaire was dispatched, $63(23 \%)$ were working, $167(60 \%)$ retired, and 29 ( $10 \%)$ unable to work.

\section{Patient symptoms}

Two hundred and seventy two (98\%) of 278 who completed all of the SF-36 completed the Rose angina questionnaire. Thirty four (13\%) reported grade 1 angina (chest pain produced on walking uphill or hurrying) and $28(10 \%)$ grade 2 (chest pain on walking at ordinary pace on level ground). Thirty eight (14\%) had "possible angina" (chest pain on exertion failing to satisfy criteria for "definite angina"), 51 (18\%) had non-exertional chest pain, and 121 (45\%) reported no problems with chest pain. Of those with exertional pain, $32(32 \%)$ reported pain less than once a week, 37 (37\%) once or twice, and $30(30 \%)$ more than twice a week.

Two hundred and seventy three (99\%) responders completed the dyspnoea scale, of which $108(40 \%)$ patients were breathless on simple exertion, $54(20 \%)$ on more strenuous exertion, and $111(41 \%)$ were not breathless even on strenuous activities.

\section{Quality of life scores}

Table 1 shows mean and median summary scores for the SF36 and SF-12. There was no significant difference between physical summary scores generated from the SF-36 and from the SF-12, whether analysed individually or as a group (paired Wilcoxon sign rank test $Z=1.83, p=0.067$ ). Individual mental scores did differ, but group scores did not.

The SF-12 detected differences in quality of life for patients with differing degrees of breathlessness and severity and frequency of angina similar to those reported previously. ${ }^{4}$

\section{DISCUSSION}

The SF-36 provides important quantitative information about the impact of disease on HRQL and is sensitive enough to identify differences in severity of post-infarction chest pain and breathlessness, readily identifying those with impaired physical or mental health who warrant physician review. ${ }^{4}$

Nevertheless, faced with increasing clinic referrals, National Service Framework targets and audits, rising patient expectations, and protocol-driven care plans, clinicians may feel disinclined to ascertain what bothers patients most, namely impaired quality of life as a result of illness. This is

Abbreviations: $H R Q L$, health related quality of life; NHP, Nottingham health profile; SF-12, short form 12 questionnaire; SF-36, short form 36 questionnaire 
Table 1 Comparison of SF-36 and SF-12 physical and mental component summary scores in a cohort of survivors of a myocardial infarction $(n=278)$

\begin{tabular}{llllll}
\hline & \multicolumn{2}{l}{ Physical component summary } & & \multicolumn{2}{l}{ Mental component summary } \\
\cline { 2 - 3 } & SF-36 & SF-12 & & SF-36 & SF-12 \\
\hline Mean & 40.42 & 40.90 & 50.52 & 49.14 \\
SD & 12.56 & 11.66 & 10.50 & 10.58 \\
Median & 41.64 & 42.28 & & 53.57 & 51.68 \\
IQR & $(30.05-51.58)$ & $(30.77-52.03)$ & & $(44.02-58.31)$ & $(40.68-57.95)$ \\
Maximum value & 63.30 & 62.37 & & 71.36 & 67.41 \\
Minimum value & 14.37 & 14.68 & 19.96 & 20.65 \\
\hline \multirow{2}{*}{ IQR, interquartile range; SD, standard deviation; SF, short form. }
\end{tabular}

not helped by extremely detailed questionnaires that are time consuming and administratively difficult, so any technique that achieves the same objective but in a more user and physician friendly way should make HRQL assessment more practical for routine use.

Utilising specific items from the SF-36 battery produces an abbreviated questionnaire, the SF-12, which has several benefits. Its brevity should increase its attractiveness to clinicians as well as patient response rates. It appears to perform well, retaining the power of the complete SF-36 to discriminate severity and frequency of exertional and nonexertional chest pain and breathlessness, despite capturing limited information. It appears to be an adequate substitute for the SF-36, retaining similar clinical discriminatory power while removing redundant questions. It remains to be established whether the SF-12 is able to detect trends over time.

There are advantages in using the SF-36 in full as we have previously shown. ${ }^{4}$ The eight domains provide more important quantitative information about the impact of disease on health related quality of life than the abbreviated form, particularly an individual's mental problems that the SF-12 might miss.

HRQL questionnaires provide a valuable and complementary insight into patients' perspective of the impact of disease on daily life. Our findings suggest that the SF-12 appears to be a robust and adequate substitute for the SF-36, capturing sufficient data to generate information equivalent to the SF36 , at least when applied to a cohort of myocardial infarction survivors. If large scale epidemiological and intervention studies require some generic measure of HRQL, the simple design of the SF-12 has distinct advantages over the more complex SF-36.

\section{Authors' affiliations}

M R Melville, M A Lari, D Gray, Cardiovascular Medicine, University Hospital, Nottingham, UK

N Brown, Royal Gwen Hospital, Newport, Gwent, UK

T Young, Health Economy Research Group, Brunel University, Uxbridge, Middlesex, UK

Correspondence to: Dr David Gray, Division of Cardiovascular Medicine, University Hospital, Nottingham NG7 2UH, UK; d.gray@nottingham.ac.uk

Accepted 7 May 2003

\section{REFERENCES}

1 Ware JE Jr, Sherbourne CD. The MOS 36-item short-form health survey (SF36). I.Conceptual framework and item selection. Med Care 1992;30:473-83.

2 Schlenk EA, Erlen JA, Dunbar-Jacob J, et al. Health-related quality of life in chronic disorders: a comparison across studies using the MOS SF-36. Qual Life Res 1998;7:57-65.

3 Jenkinson C, Coulter A, Wright L. Short form 36 (SF36) health survey questionnaire: normative data for adults of working age. $B M J$ $1993 \cdot 306: 1437-40$
4 Brown N, Melville M, Gray D, et al. Quality of life four years after acute myocardial infarction: short form 36 scores compared with a normal population. Heart 1999;81:352-8.

5 Brown N, Melville M, Gray D, et al. Comparison of the SF-36 health survey questionnaire with the Nottingham Health Profile in long-term survivors of a myocardial infarction. J Public Health Med 2000;22:167-75.

\section{WEB TOP 10}

www.heartinl.com

These articles scored the most hits on Heart's website during September 2003

1 Atrial fibrillation: classification, pathophysiology, mechanisms and drug treatment

$\checkmark$ Markides, $R$ J Schilling

August 2003;89:939-43. (Education in Heart)

2 Inflammatory and thrombotic mechanisms in coronary atherosclerosis

D Tousoulis, G Davies, C Stefanadis, P Toutouzas,

J A Ambrose

September 2003;89:993-7. (Review)

3 Basic aspects of plaque vulnerability

A Lafont

October 2003;89:1262-7. (Education in Heart)

4 Left ventricular remodelling after myocardial infarction: importance of residual myocardial viability and ischaemia C A Visser

October 2003;89:1121-2. (Editorial)

5 Plaque stabilisation in acute coronary syndromes: clinical considerations

A D Timmis

October 2003;89:1268-72. (Education in Heart)

6 Antiplatelet treatment in stable coronary artery disease

C J Knight

October 2003;89:1273-78. (Education in Heart)

7 Practical aspects of the management of pericardial disease

B Maisch, A D Ristic

September 2003;89:1096-103. (Education in Heart)

8 Cardiovascular malformations in infants of diabetic mothers

C Wren, G Birrell, G Hawthorne

October 2003;89:1217-20. (Congenital heart disease)

9 Off-pump coronary artery bypass graft surgery: the incidence of postoperative atrial fibrillation

R A Archbold, N P Curzen

October 2003;89:1 134-7. (Review)

10 Joint British recommendations on prevention of coronary heart disease in clinical practice

December 2001, 1998;80:1-29.

Visit the Heart website for hyperlinks to these articles, by clicking on "Top 10 papers"

www.heartinl.com 